\title{
Solar System Motions and the Cosmological Constant: A New Approach
}

\author{
Lorenzo Iorio \\ INFN-Sezione di Pisa, 56127 Pisa, Italy \\ Correspondence should be addressed to Lorenzo Iorio, lorenzo.iorio@libero.it \\ Received 11 October 2007; Revised 17 January 2008; Accepted 12 February 2008 \\ Recommended by Edmond Craig Dukes
}

\begin{abstract}
We use the corrections to the Newton-Einstein secular precessions of the longitudes of perihelia $\dot{\omega}$ of some planets (Mercury, Earth, Mars, Jupiter, Saturn) of the Solar System, phenomenologically estimated as solve-for parameters by the Russian astronomer E. V. Pitjeva in a global fit of almost one century of data with the EPM2004 ephemerides, in order to put on the test the expression for the perihelion precession induced by a uniform cosmological constant $\Lambda$ in the framework of the Schwarzschild-de Sitter (or Kottler) space-time. We compare such an extra rate to the estimated corrections to the planetary perihelion precessions by taking their ratio for different pairs of planets instead of using one perihelion at a time for each planet separately, as done so far in literature. The answer is negative, even by further rescaling by a factor 10 (and even 100 for Saturn) the errors in the estimated extra precessions of the perihelia released by Pitjeva. Our conclusions hold also for any other metric perturbation having the same dependence on the spatial coordinates, as those induced by other general relativistic cosmological scenarios and by many modified models of gravity. Currently ongoing and planned interplanetary spacecraft-based missions should improve our knowledge of the planets' orbits allowing for more stringent constraints.
\end{abstract}

Copyright ( $\odot 2008$ Lorenzo Iorio. This is an open access article distributed under the Creative Commons Attribution License, which permits unrestricted use, distribution, and reproduction in any medium, provided the original work is properly cited.

\section{Introduction}

Introduced for the first time by Einstein [1] to allow static homogeneous solutions to Einstein's equations in the presence of matter, the cosmological constant $\Lambda$, which turned out to be unnecessary after the discovery of the cosmic expansion by Hubble [2], has been recently brought back mainly as the simplest way to accommodate, in the framework of general relativity, the vacuum energy needed to explain the observed acceleration of the universe $[3,4]$. For the relation between the cosmological constant and the dark energy see [5]. For a general overview of the cosmological constant see [6] and references therein. Theoretical problems concerning the cosmological constant are reviewed in [7].

Since, at present, there are no other independent signs of the existence of $\Lambda$ apart from the cosmological acceleration itself, attempts were made in the more or less recent past to find evidence of it in phenomena occurring on local, astronomical scales with particular emphasis on the precession of the perihelia $\omega$ of the Solar System's inner planets [8-20] (for other local effects induced by $\Lambda$ like gyroscope precession, precession, mean motion change, geodetic precession, gravitational red-shift, deflection of light, gravitational time-delay, and doppler tracking of spacecraft on escape trajectories, see, e.g., $[15,18])$.

\section{The Perihelion Precession Induced by a Uniform Cosmological Constant and the Confrontation with the Data}

Starting from the radial acceleration [21]

$$
\mathbf{A}_{\Lambda}=\frac{1}{3} \Lambda c^{2} \mathbf{r}
$$

where $c$ is the speed of light, imparted by a uniform cosmological constant $\Lambda$ in the framework of the spherically symmetric Schwarzschild vacuum solution with a cosmological constant, that is the Schwarzschild-de Sitter [22] or Kottler [23] space-time, Kerr et al. [16] by using the Gauss equations for the variation of the Keplerian orbital elements [24] worked out the secular, that is, averaged over one orbital 
revolution, precession of the pericenter of a test-body induced by the cosmological constant $\Lambda$ finding

$$
\langle\dot{\omega}\rangle_{\Lambda}=\frac{1}{2}\left(\frac{\Lambda c^{2}}{n}\right) \sqrt{1-e^{2}}
$$

where $n=\sqrt{G M / a^{3}}$ is the Keplerian mean motion of the planet moving around a central body of mass $M, G$ is the Newtonian constant of gravitation, and $a$ and $e$ are the semimajor axis and the eccentricity, respectively, of the testbody's orbit.

Here, we wish to offer an alternative derivation of (2) based on the use of the Lagrange perturbative scheme [24]. The Lagrange equation for the pericenter is

$$
\frac{d \omega}{d t}=\frac{1}{n a^{2} \sqrt{1-e^{2}} \tan i} \frac{\partial\left\langle\mathcal{V}_{\text {pert }}\right\rangle}{\partial i}-\frac{\sqrt{1-e^{2}}}{n a^{2} e} \frac{\partial\left\langle\mathcal{V}_{\text {pert }}\right\rangle}{\partial e},
$$

where $i$ is the inclination angle to the equator of the central mass and $\left\langle\mathcal{V}_{\text {pert }}\right\rangle$ is the perturbing potential $\mathcal{V}_{\text {pert }}$ averaged over one orbital revolution. For the Schwarzschild-de Sitter space-time, the cosmologically induced additional potential is [16]

$$
\mathcal{V}_{\Lambda}=-\frac{1}{16} \Lambda c^{2} r^{2}
$$

By evaluating (4) onto the unperturbed Keplerian ellipse defined by

$$
r=a(1-e \cos E)
$$

where $E$ is the eccentric anomaly, and integrating over one orbital period $P_{b}=2 \pi / n$ by means of

$$
d t=\left(\frac{1-e \cos E}{n}\right) d E,
$$

the result

$$
\int_{0}^{2 \pi}(1-e \cos E)^{3} d E=\pi\left(2+3 e^{2}\right)
$$

yields

$$
\left\langle\mathcal{V}_{\Lambda}\right\rangle=-\frac{1}{12} \Lambda c^{2} a^{2}\left(2+3 e^{2}\right)
$$

By inserting (8) into (3), one obtains just (2).

Jetzer and Sereno [14], Sereno and Jetzer [19], Adkins et al. [8] and Adkins and McDonnell [9] obtained, in different frameworks, the same result of (2). Note that $\langle\dot{\omega}\rangle_{\Lambda} \propto$ $\sqrt{a^{3}\left(1-e^{2}\right)}$, where, for a uniform $\Lambda$, the proportionality factor is common to all the bodies orbiting a given central mass. Moreover, (2) was obtained by using the standard radial isotropic coordinate which is commonly used in the Solar System planetary data reduction process to produce the ephemerides [25], so that (2) can meaningfully be used for comparisons with the latest observational determinations of the non-Newtonian/Einsteinian secular precessions of the longitude of the perihelia $\varpi$ (the longitude of perihelion $\varpi$ is defined, for orbits nearly equatorial like the Solar System's ones, as the sum of the argument of perihelion $\omega$ and of the longitude of the ascending node $\Omega$; the latter one, sensitive to the out-of-plane disturbing forces, is not affected by the entirely radial $\Lambda$-induced extra acceleration) [26]. Indeed, they were estimated by contrasting, in a least square sense, almost one century of data of different kinds with the suite of dynamical force models of the EPM2004 ephemerides [27] which included all the standard Newtonian and Einsteinian dynamics, apart from just any exotic effects as the ones by $\Lambda$ on both the geodesic equations of motion and of the electromagnetic waves. Thus, such extra precessions of perihelia, estimated independently of our goal, account in principle for any unmodeled force existing in nature.

Since the cosmological accelerated expansion yields $\Lambda \approx$ $10^{-56} \mathrm{~cm}^{-2}$, Kerr et al. [16] concluded that the precession of (2) is too small to be measured in the Solar System. Iorio [12], Jetzer and Sereno [14], Kagramanova et al. [15], Sereno and Jetzer [18], Sereno and Jetzer [19], Adkins et al. [8] Adkins and McDonnell [9] used (2) and the extra precessions of the inner planets of the Solar System estimated by Pitjeva [26] to put constraints on $\Lambda$. In particular, Jetzer and Sereno [14], after working out the effect of $\Lambda$ on the pericenter of a general two-body system with arbitrary masses in the standard post-Newtonian gauge, used various binary pulsar systems and planets of the Solar System one at a time separately; Sereno and Jetzer [18] discussed the possibilities offered by future interplanetary ranging, especially for Pluto; Sereno and Jetzer [19] and Adkins et al. [8] obtained (2) as a particular case in the framework of cosmological models with a nonnull acceleration. Adkins and McDonnell [9] worked out the perihelion precessions under the action of arbitrary central forces obtaining (2) as a particular case. In all such cases-and also in previous analyses performed when only data for Mercury existed such as $[10,13,17,20]$ (in fact, also the non-Newtonian perihelion precession of the highly eccentric $(e=0.826)$ orbit of the asteroid Icarus was investigated [28-31], but it was never used for putting constraints on $\Lambda$. We will not use it in the present analysis) the general scheme followed was to assume the planets, or the pulsar systems considered, separately one at a time and to derive constraints on $\Lambda$ for each of them by considering it as a free parameter.

\section{Taking the Ratio of the Perihelia}

Here we will follow, instead, a different approach which is able to tell us something much more definite about (1) and (2). We will construct the ratios of the estimated extra precessions of perihelion for different pairs of planets A and $\mathrm{B}$ and will compare them to the corresponding ratios of the precessions of (2) for the same planets. Thus, it is possible to construct the following quantity:

$$
\lambda_{\mathrm{AB}}=\left|\frac{\dot{\omega}_{\mathrm{A}}}{\dot{\omega}_{\mathrm{B}}}-\sqrt{\frac{a_{\mathrm{A}}^{3}\left(1-e_{\mathrm{A}}^{2}\right)}{a_{\mathrm{B}}^{3}\left(1-e_{\mathrm{B}}^{2}\right)}}\right| .
$$

If (2) is correct, independently of the value of $\Lambda$ provided that, of course, it is nonzero and small enough to assure 
TABLE 1: Estimated semimajor axes $a$, in $\mathrm{AU}\left(1 \mathrm{AU}=1.49597870691 \times 10^{11} \mathrm{~m}\right)$ [27], and phenomenologically estimated corrections to the Newtonian-Einsteinian perihelion rates, in arcseconds per century $\left({ }^{\prime \prime} \mathrm{cy}^{-1}\right)$, of Mercury, the Earth, and Mars [26]. Also the associated errors are quoted: they are in $\mathrm{m}$ for $a$ [27] and in "cy ${ }^{-1}$ for $\dot{\boldsymbol{\omega}}$ [26]. For the semimajor axes, they are the formal, statistical ones, while for the perihelia, they are realistic in the sense that they were obtained from comparison of many different solutions with different sets of parameters and observations [35]. However, the results presented in the text do not change if $\delta \dot{\omega}$ are rescaled by a factor 10.

\begin{tabular}{lcccc}
\hline Planet & $a(\mathrm{AU})$ & $\delta a(\mathrm{~m})$ & $\dot{\omega}\left({ }^{\prime \prime} \mathrm{cy}^{-1}\right)$ & $\delta \dot{\omega}\left(" \mathrm{cy}^{-1}\right)$ \\
\hline Mercury & 0.38709893 & 0.105 & -0.0036 & 0.0050 \\
Earth & 1.00000011 & 0.146 & -0.0002 & 0.0004 \\
Mars & 1.52366231 & 0.657 & 0.0001 & 0.0005 \\
\hline
\end{tabular}

TABLE 2: Estimated semimajor axes $a$, in $\mathrm{AU}\left(1 \mathrm{AU}=1.49597870691 \times 10^{11} \mathrm{~m}\right)$ [27], and phenomenologically estimated corrections to the Newtonian-Einsteinian perihelion rates [35], in arcseconds per century $\left({ }^{\prime \prime} \mathrm{cy}^{-1}\right)$, of Jupiter, Saturn, and Uranus. Also the associated errors are quoted: they are in $\mathrm{m}$ for $a$ [27] and in "cy ${ }^{-1}$ for $\dot{\omega}$ [35]. For the semimajor axes, they are the formal, statistical ones, while for the perihelia, they are the formal ones rescaled by a factor 10 in order to yield realistic estimates for them.

\begin{tabular}{lcccc}
\hline Planet & $a(\mathrm{AU})$ & $\delta a(\mathrm{~m})$ & $\dot{\omega}\left({ }^{\prime c} \mathrm{cy}^{-1}\right)$ & $\delta \dot{\omega}\left(" \mathrm{cy}^{-1}\right)$ \\
\hline Jupiter & 5.20336301 & 639 & 0.0062 & 0.036 \\
Saturn & 9.53707032 & 4222 & -0.92 & 2.9 \\
Uranus & 19.19126393 & 38484 & 0.57 & 13 \\
\hline
\end{tabular}

that the perturbation approach followed to derive (2) is appropriate, then (9) must be compatible with zero within the errors in $\dot{\boldsymbol{\omega}}$ and $a$ [27]. It must be noted that such an approach holds, in general, also for any other extra acceleration term, whatever origin it may have, of the form

$$
\mathbf{A}=\mathfrak{K} \mathbf{r}, \quad \mathfrak{K} \neq 0,
$$

so that our conclusions will not be restricted to the Schwarzschild-de Sitter space-time only. Indeed, there are other general relativistic cosmological models [32] and many long-range models of modified gravity [33] which are able to induce a perturbing acceleration like that of (10) [34].

\subsection{The Inner Planets}

Let us start with the inner planets whose relevant orbital parameters are listed in Table 1 . The answer they give is negative. Indeed, for the pairs $\mathrm{A}=$ Mars, $\mathrm{B}=$ Mercury and $\mathrm{A}=$ Earth, $\mathrm{B}=$ Mercury we have

$$
\begin{aligned}
\lambda_{\text {MarMer }} & =7.8 \pm 0.2, \\
\lambda_{\text {EarMer }} & =4.1 \pm 0.2 ;
\end{aligned}
$$

a negative result at $40-\sigma$ and $20-\sigma$ levels, respectively. The other four pairs of inner planets yield, instead, results compatible with zero. The uncertainties in (11) have been conservatively worked out by propagating the errors (the eccentricities are negligible) in $\dot{\omega}$ and $a$ in (9) and linearly summing the resulting biased terms

$$
\delta \lambda_{\mathrm{AB}} \leq\left|\frac{\dot{\omega}^{\mathrm{A}}}{\dot{\omega}^{\mathrm{B}}}\right|\left(\frac{\delta \dot{\omega}^{\mathrm{A}}}{\left|\dot{\omega}^{\mathrm{A}}\right|}+\frac{\delta \dot{\omega}^{\mathrm{B}}}{\left|\dot{\omega}^{\mathrm{B}}\right|}\right)+\frac{3}{2}\left(\frac{a^{\mathrm{A}}}{a^{\mathrm{B}}}\right)^{3 / 2}\left(\frac{\delta a^{\mathrm{A}}}{a^{\mathrm{A}}}+\frac{\delta a^{\mathrm{B}}}{a^{\mathrm{B}}}\right)
$$

the dominant sources of uncertainty are by far the perihelion rates. It is important to stress that even if $\delta \dot{\omega}$ were 10 times larger than the errors released by Pitjeva [26] and reproduced in Table 1-which are not the mere formal, statistical oneswe would still be forced to rule out (2) at some $\sigma$ level.

\subsection{The Outer Planets}

It is interesting to use also some of the outer planets for which it was possible to estimate the corrections to the perihelion precessions [35], quoted in Table 2. The giant planets whose extra precessions of the perihelion are at our disposal are Jupiter, Saturn, and Uranus because the temporal extension of the data set used covered at least one full orbital revolution just for such planets: indeed, the orbital periods of Neptune and Pluto amount to about 164 and 248 years, respectively. For the external regions of the Solar System only optical observations were used, apart from Jupiter [27]; they are, undoubtedly, of poorer accuracy with respect to those used for the inner planets which also benefit of radar-ranging measurements, but we will show that they are accurate enough for our purposes. Let us stress that in Table 2 we rescaled by a factor 10 the otherwise formal, statistical errors in the estimated extra rates of perihelia. The pair $\mathrm{A}=$ Jupiter, B = Saturn yields

$$
\lambda_{\text {JupSat }}=0.41 \pm 0.06 ;
$$

it is incompatible with zero at about $7-\sigma$ level. If we further rescale by 10 the error of Table 2 in the extra precession of Saturn, for which Pitjeva did not use radiometric data, that is, by 100 its formal, statistical error, we get

$$
\lambda_{\text {JupSat }}^{\prime}=0.4 \pm 0.2,
$$

negative at $2-\sigma$ level. In regard to Jupiter, since Pitjeva [27] used also some radiometric data for it, we believe that a rescaling of 10 of the formal error in its estimated perihelion extra rate is adequate. 
For the pair $\mathrm{A}=$ Jupiter, $\mathrm{B}=$ Mercury we have

$$
\lambda_{\text {JupMer }}=51 \pm 12
$$

by using the figure of Table 1 for the uncertainty in the extra precession of Mercury; a negative result at more than $4-\sigma$ level. If, in a very pessimistic approach, we rescale by 10 the error of Table 1 in Mercury's extra rate-although it is not the formal one-we get

$$
\lambda_{\text {JupMer }}^{\prime}=51 \pm 34 \text {, }
$$

which is incompatible with zero at $1.5-\sigma$ level. The errors in the semimajor axes, even if rescaled by 10 or more, do not affect at all our results. The other pairs of planets yield results compatible with zero.

\section{Conclusions}

In this paper, we used the latest observation-based determinations of the non-Newtonian/Einsteinian precessions of the longitudes of perihelia $\dot{\dot{\omega}}$ of some planets of the Solar System obtained by E. V. Pitjeva (Institute of Applied Astronomy, Russian Academy of Sciences) [26, 35] with the EPM ephemerides [27] to constrain the dynamical effects induced in our planetary arena by a nonzero uniform cosmological constant in the framework of the Schwarzschild-de Sitter (or Kottler) space-time. Such corrections to the standard perihelion precessions were determined without modeling at all the effects of $\Lambda$ on both the geodesic equations of motion of planets and electromagnetic waves carrying information on them, so that they fully account, in principle, for such effects. The ratios of different pairs of planetary perihelia were used; by conservatively treating the errors in the estimated extra precessions, it turns out that the expression of the $\Lambda$-induced perihelion precession is ruled out at many $\sigma$ levels. It is important to note that our phenomenological approach is quite general, because it holds also for any small extra acceleration of the form $\mathbf{A}=\mathfrak{K} \mathbf{r}, \mathfrak{K} \neq 0$ : such a functional form is, in fact, induced not only by general relativistic cosmological models [32] but also by modified models of gravity (see, e.g., $[33,34]$ ).

The present analysis relies only upon the extra precessions estimated so far by Pitjeva; it would be useful if also other teams of astronomers would estimate independently their own corrections to the standard planetary perihelion rates by exploiting the huge records of modern observations currently available. In regard to future perspectives, our knowledge of the motion of the inner planets of the Solar System should improve in the near future thanks to the ongoing Messenger (http://messenger.jhuapl.edu/) and Venus Express (VEX) [36] missions to Mercury and Venus, respectively, and to the planned BepiColombo (http://messenger.jhuapl.edu/) [37] mission to Mercury; also planetary laser ranging (PLR) [38], for example, to a lander on Mars [39] or to the Mercury laser altimeter [40], would greatly increase the accuracy in planetary orbit determination. In regard to the outer regions of the Solar System, the processing of the radiometric data from the ongoing Saturnian mission of Cassini and from Jupiter's flyby of New Horizons available at http://pluto.jhuapl.edu/ (it took a gravity assist by Jupiter in February 2007 and should reach the orbit of Saturn in mid2008), recently occurred, might improve our knowledge of the motion of the outer planets as well in a not too far future.

\section{Acknowledgment}

The author gratefully thanks E. V. Pitjeva for her information about the perihelion precessions of the outer planets of the Solar System.

\section{References}

[1] A. Einstein, "Kosmologische betrachtungen zur allgemeinen relativitätstheorie," Sitzungsberichte der Königlich Preußischen Akademie der Wissenschaften, pp. 142-152, 1917.

[2] E. Hubble, "A relation between distance and radial velocity among extra-galactic nebulae," Proceedings of the National Academy of Sciences of the United States of America, vol. 15, no. 3, pp. 168-173, 1929.

[3] S. Perlmutter, G. Aldering, G. Goldhaber, et al., "Measurements of $\Omega$ and $\Lambda$ from 42 high-redshift supernovae," The Astrophysical Journal, vol. 517, no. 2, pp. 565-586, 1999.

[4] A. G. Riess, A. V. Filippenko, P. Challis, et al., "Observational evidence from supernovae for an accelerating universe and a cosmological constant," The Astronomical Journal, vol. 116, no. 3, pp. 1009-1038, 1998.

[5] P. J. Peebels and B. Ratra, "The cosmological constant and dark energy," Reviews of Modern Physics, vol. 75, no. 2, pp. 559-606, 2003.

[6] S. M. Carroll, "The cosmological constant," Living Reviews in Relativity, vol. 4, no. 1, pp. 1-78, 2001.

[7] S. Weinberg, "The cosmological constant problem," Reviews of Modern Physics, vol. 61, no. 1, pp. 1-23, 1989.

[8] G. S. Adkins, J. McDonnell, and R. N. Fell, "Cosmological perturbations on local systems," Physical Review D, vol. 75, no. 6, Article ID 064011, 9 pages, 2007.

[9] G. S. Adkins and J. McDonnell, "Orbital precession due to central-force perturbations," Physical Review D, vol. 75, no. 8, Article ID 082001, 7 pages, 2007.

[10] J. F. Cardona and J. M. Tejeiro, "Can interplanetary measures bound the cosmological constant?" The Astrophysical Journal, vol. 493, pp. 52-53, 1998.

[11] Yu. V. Dumin, "Testing the "dark-energy"-dominated cosmology via the solar-system experiments," preprint, 2006, http://arxiv.org/abs/astro-ph/0507381.

[12] L. Iorio, "Can solar system observations tell us something about the cosmological constant?" International Journal of Modern Physics D, vol. 15, no. 4, pp. 473-475, 2006.

[13] J. N. Islam, "The cosmological constant and classical tests of general relativity," Physics Letters A, vol. 97, no. 6, pp. 239-241, 1983.

[14] Ph. Jetzer and M. Sereno, "Two-body problem with the cosmological constant and observational constraints," Physical Review D, vol. 73, no. 4, Article ID 044015, 6 pages, 2006.

[15] V. Kagramanova, J. Kunz, and C. Lämmerzahl, "Solar system effects in Schwarzschild-de Sitter space-time," Physics Letters $B$, vol. 634, no. 5-6, pp. 465-470, 2006.

[16] A. W. Kerr, J. C. Hauck, and B. Mashhoon, "Standard clocks, orbital precession and the cosmological constant," Classical and Quantum Gravity, vol. 20, no. 13, pp. 2727-2736, 2003.

[17] G. V. Kraniotis and S. B. Whitehouse, "Compact calculation of the perihelion precession of mercury in general relativity, 
the cosmological constant and Jacobi's inversion problem," Classical and Quantum Gravity, vol. 20, no. 22, pp. 4817-4835, 2003.

[18] M. Sereno and Ph. Jetzer, "Solar and stellar system tests of the cosmological constant," Physical Review D, vol. 73, no. 6, Article ID 063004, 5 pages, 2006.

[19] M. Sereno and Ph. Jetzer, "Evolution of gravitational orbits in the expanding universe," Physical Review D, vol. 75, no. 6, Article ID 064031, 8 pages, 2007.

[20] E. L. Wright, "Interplanetary measures can not bound the cosmological constant," preprint, 1998, http://arxiv.org/abs/ astro-ph/9805292v1.

[21] W. Rindler, Relativity: Special, General, and Cosmological, Oxford University Press, Oxford, UK, 2001.

[22] Z. Stuchlík and S. Hledík, "Some properties of the Schwarzschild-de Sitter and Schwarzschild-anti-de Sitter spacetimes," Physical Review D, vol. 60, no. 4, Article ID 044006, 15 pages, 1999.

[23] F. Kottler, "Über die physikalischen grundlagen der Einsteinschen gravitationstheorie," Annalen der Physik, vol. 361, no. 14 , pp. 401-462, 1918.

[24] A. E. Roy, Orbital Motion, Institute of Physics, Bristol, UK, 4th edition, 2005.

[25] F. Estabrook, JPL IOM, Section 328, 1-3, 1971.

[26] E. V. Pitjeva, "Relativistic effects and solar oblateness from radar observations of planets and spacecraft," Astronomy Letters, vol. 31, no. 5, pp. 340-349, 2005.

[27] E. V. Pitjeva, "High-precision ephemerides of planets-EPM and determination of some astronomical constants," Solar System Research, vol. 39, no. 3, pp. 176-186, 2005.

[28] B. Shahid-Saless and D. K. Yeomans, "Relativistic effects on the motion of asteroids and comets," The Astronomical Journal, vol. 107, no. 5, pp. 1885-1889, 1994.

[29] I. I. Shapiro, W. B. Smith, M. E. Ash, and S. Herrick, "General relativity and the orbit of Icarus," The Astronomical Journal, vol. 76, no. 7, pp. 588-606, 1971.

[30] G. Sitarski, "On the relativistic motion of (1566) Icarus," The Astronomical Journal, vol. 104, no. 3, pp. 1226-1229, 1992.

[31] J.-X. Zhang, "A study of general relativistic effect in the motion of Icarus," Chinese Astronomy and Astrophysics, vol. 18, no. 1, pp. 108-115, 1994.

[32] B. Mashhoon, N. Mobed, and D. Singh, "Tidal dynamics in cosmological spacetimes," Classical and Quantum Gravity, vol. 24, no. 20, pp. 5031-5046, 2007.

[33] G. Cognola, E. Elizalde, S. Nojiri, S. D. Odintsov, and S. Zerbini, "One-loop $f(R)$ gravity in de Sitter universe," Journal of Cosmology and Astroparticle Physics, vol. 2005, no. 2, p. 10, 2005.

[34] M. L. Ruggiero and L. Iorio, "Solar system planetary orbital motions and $f(R)$ theories of gravity," Journal of Cosmology and Astroparticle Physics, vol. 1, no. 10, 2007.

[35] E. V. Pitjeva, private communication, 2006.

[36] A. Fienga, H. Manche, J. Laskar, and M. Gastineau, "INPOP06: a new numerical planetary ephemeris," Astronomy \& Astrophysics, vol. 477, no. 1, pp. 315-327, 2008.

[37] A. Milani, D. Vokrouhlický, D. Villani, C. Bonanno, and A. Rossi, "Testing general relativity with the BepiColombo radio science experiment," Physical Review D, vol. 66, no. 8, Article ID 082001, 21 pages, 2002.

[38] J. F. Chandler, M. R. Pearlman, R. D. Reasenberg, and J. J. Degnan, "Solar-system dynamics and tests of general relativity with planetary laser ranging," in Proceedings of the 14th
International Workshop on Laser Ranging Instrumentation, R. Noomen, J. M. Davila, J. Garate, C. Noll, and M. Pearlman, Eds., San Fernando, Spain, June 2004.

[39] S. M. Merkowitz, P. W. Dabney, J. C. Livas, J. F. McGarry, G. A. Neumann, and T. W. Zagwodzki, "Laser ranging for gravitational, lunar and planetary science," International Journal of Modern Physics D, vol. 16, no. 12, pp. 2151-2164, 2007.

[40] X. Sun, G. A. Neumann, J. F. Mc-Garry, et al., "Laser ranging between the mercury laser altimeter and an earth-based laser satellite tracking station over a 24 million kilometer distance," in OSA Annual Meeting Abstracts, Tucson, Ariz, USA, October 2005. 

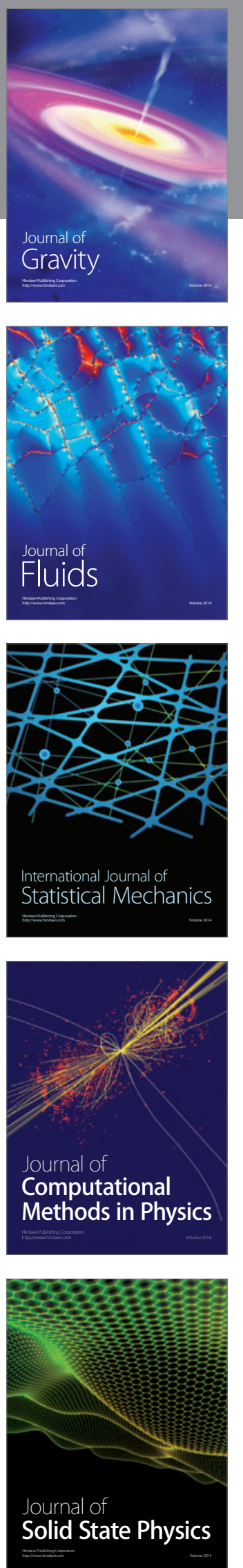

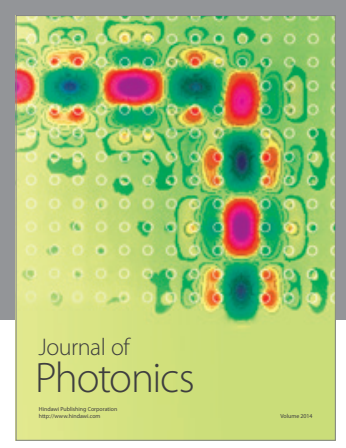

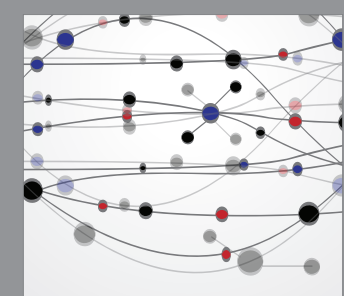

The Scientific World Journal
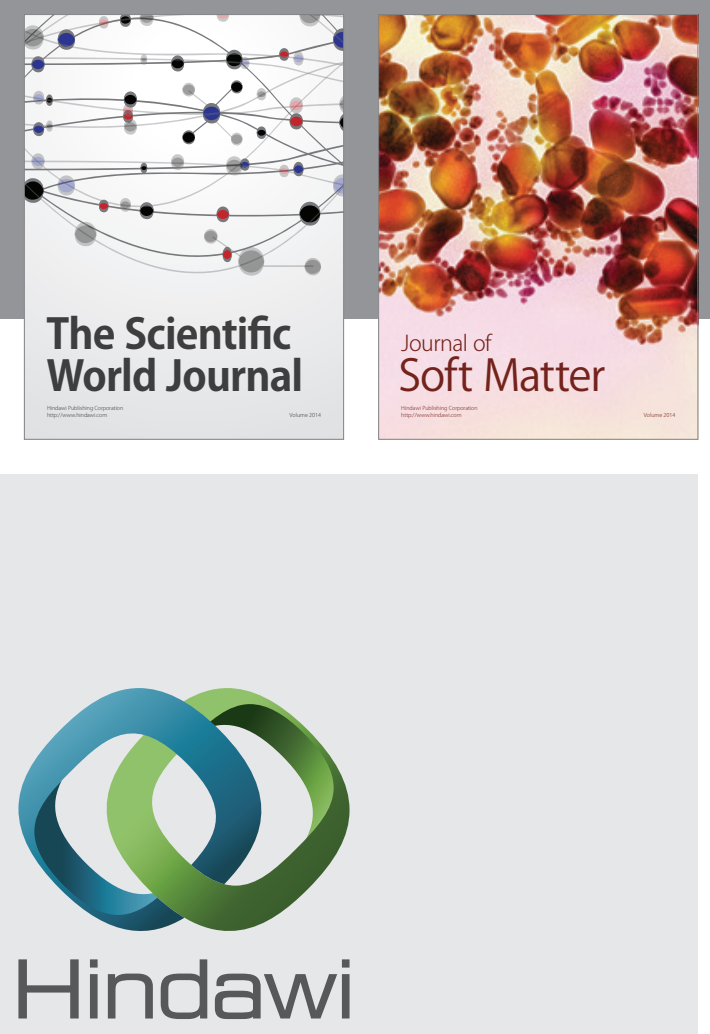

Submit your manuscripts at

http://www.hindawi.com
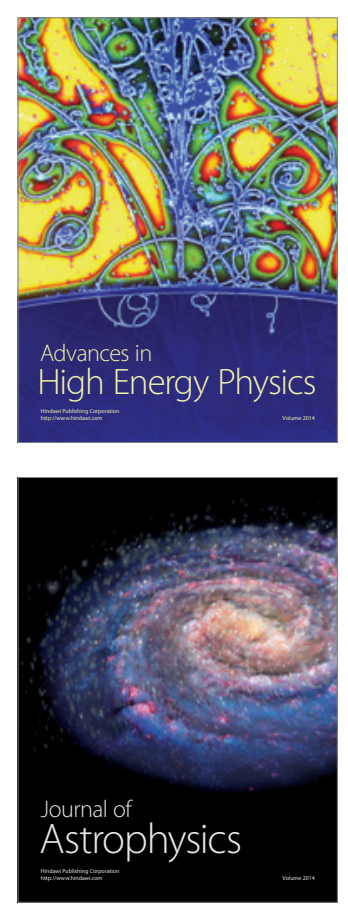
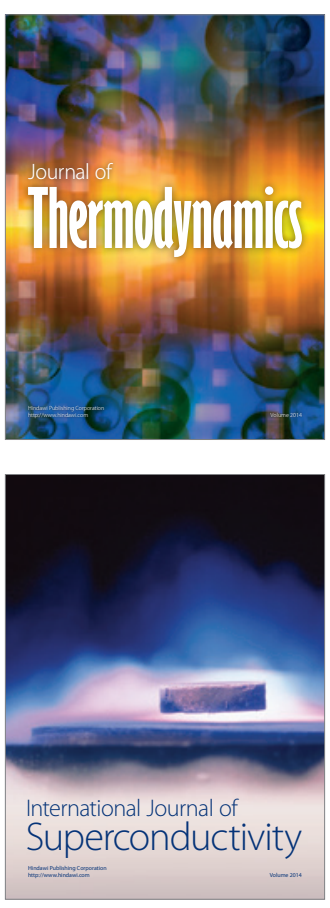
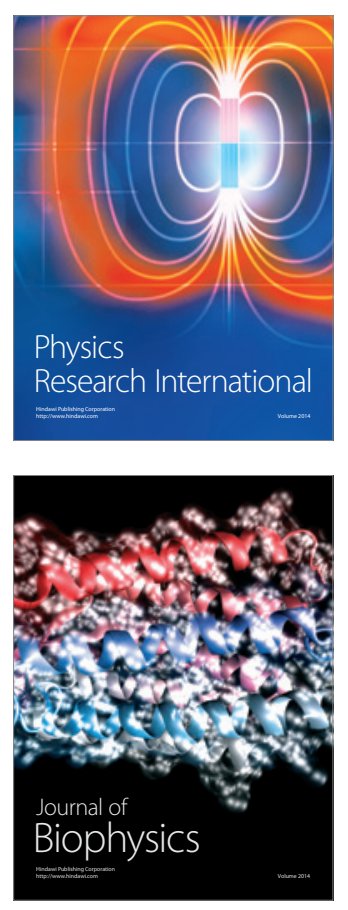
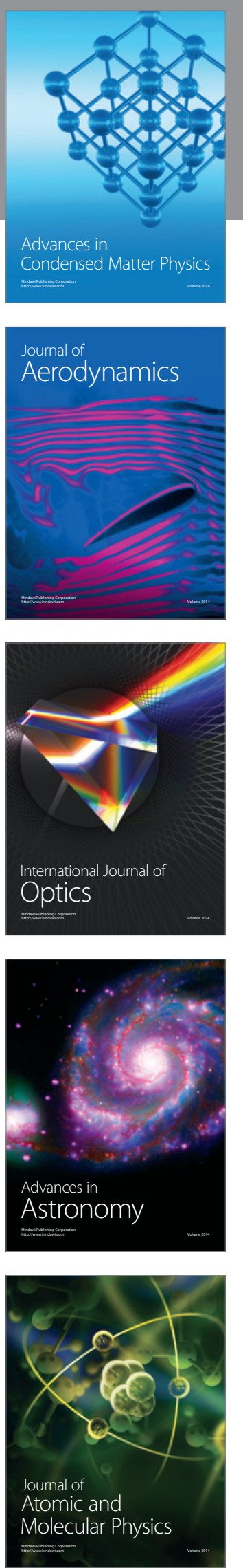